\title{
Screen printed carbon electrodes incorporating electrochemical molecularly imprinted polymers to detect pollutant
}

\author{
Vitalys Mba Ekomo ${ }^{1}$, Catherine Branger ${ }^{1}$, Raphaël Bikanga ${ }^{2}$, Georges Istamboulie ${ }^{3,4}$, Carole Calas- \\ Blanchard $^{3,4}$, Thierry Noguer ${ }^{3,4}$, Huques Brisset $^{1}$ \\ ${ }^{1}$ Université de Toulon, Laboratoire MAPIEM, Toulon, France \\ ${ }^{2}$ Laboratoire de Substances naturelles et de Synthèse organique, Université des Sciences et \\ Techniques de Masuku, B.P. 901 Franceville, Gabon \\ ${ }^{3}$ Univ. Perpignan Via Domitia, Biocapteurs-Analyses-Environnement, 66860 Perpignan, France \\ ${ }^{4}$ Laboratoire de Biodiversité et Biotechnologies Microbiennes, USR 3579 Sorbonne Universités \\ (UPMC) Paris 6 et CNRS Observatoire Océanologique, 66650 Banyuls-sur-Mer, France
}

\author{
brisset@univ-tIn.fr
}

\begin{abstract}
:
Electrochemical molecularly imprinted polymers (e-MIP) are MIP specially designed to detect organic molecules thanks to the electrochemical answer of a redox probe inserted inside the binding cavities of cross-linked MIPs. Herein, e-MIP microbeads particles were synthesized from ferrocenylmethyl methacrylate and 4-vinylpyridine functional co-monomers, bisphenol A (BPA) as the target and ethylene glycol dimethacrylate as cross-linker. In addition of the role of monomer, ferrocenylmethyl methacrylate acts as a redox probe enabling the electrochemical detection of the target. After physicochemical characterization, e-MIP microbeads were incorporated in a graphite-hydroxyethylcellulose composite paste to prepare screen-printed carbon electrodes (SPCE). Electrochemical properties of eMIP-SPCE revealed a highly sensitivity in the presence of BPA in aqueous medium compared to the corresponding non imprinted polymer (e-NIP-SPCE) with a limit of detection (LOD) of $0.06 \mathrm{nM}$.
\end{abstract}

Key words: molecularly imprinted polymer, ferrocene, electrochemistry, bisphenol A, screen printed electrode.

\begin{abstract}
Introduction
Molecularly Imprinted Polymers (MIPs) are three-tridimensional polymers network prepared around a molecular template. The release of template leads to polymers with binding cavities, which retain shape, size and orientation of the target molecule. Due to their simple synthesis, high stability and low cost MIPs can advantageously replace natural receptors in biosensor devices [1]. Based on this, we developed strategy to detect molecular targets, based on the introduction of a redox probe in the form of a functional monomer inside MIP microparticles [2].
\end{abstract}

Here, we present (a) the synthesis and characterization of electrochemical cross-linked molecularly imprinted polymers (e-MIP) based on ferrocenylmethyl methacrylate as functional redox tracer for Bisphenol A (BPA) determination and (b) the first electrochemical results obtained with electrochemical MIPs incorporated in a graphite-hydroxyethyl cellulose composite paste screen-printed electrode [3].

\section{Results}

Electrochemical e-MIP and e-NIP (electrochemical Non-Imprinted Polymer) were prepared by precipitation polymerization in acetonitrile with ferrocenyl methylmethacrylate and 4-vinylpyridine as co-functional monomers, ethyleneglycodimethacrylate as the crosslinker and bisphenol $A$ as the target (Figure 1). 


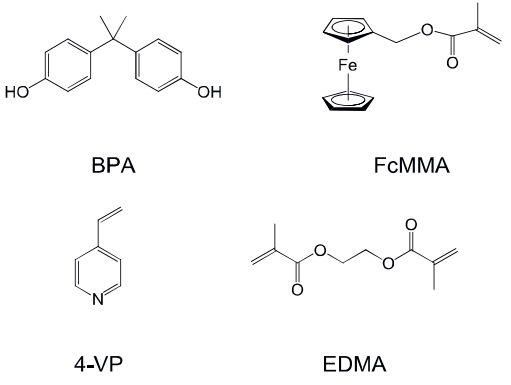

Fig. 1. Molecular structures of e-MIP components.

Characterization of polymers by FTIR spectroscopy confirmed the presence of the ferrocene moiety by its symmetric ring in plane vibration at $1003 \mathrm{~cm}^{-1}$ and ring-Fe vibration band at $484 \mathrm{~cm}^{-1}$. The correct leaching of BPA from the e-MIP was confirmed by the absence of any major characteristic band of BPA in the e-MIP spectrum and by the high similitude of the e-MIP and e-NIP spectra.

SEM pictures revealed spherical particles with average diameters of $4.40 \mu \mathrm{m}$ for the e-MIP and $3.60 \mu \mathrm{m}$ for the e-NIP with low coefficients of variation of 15 and $10 \%$ respectively

The adsorption of BPA was studied in aqueous medium by batch experiments after an equilibration time of $24 \mathrm{~h}$. The maximum binding capacity of the e-MIP is $27 \mathrm{mg} / \mathrm{g}$.

A mixture of graphite powder and hydroxyethylcellulose was used to prepare the Screen Printed Carbon Electrode (SPCE). Cyclic voltammograms (CV) of SCPE modified with e-MIP (e-MIP-SPCE) or e-NIP (e-NIPSPCE) were carried out in $0.1 \mathrm{M} \mathrm{KCl}$ aqueous solution at $25 \mathrm{mV} / \mathrm{s}$. CVs revealed a reversible redox system related to the ferricenium/ferrocene couple included in the polymers. After recording the first $\mathrm{CV}$, BPA was progressively added to raise its concentration from 0 to $413 \mathrm{ng} / \mathrm{L}$. As expected, a decrease of the peak intensity was observed for the e-MIP-SPCEs in good agreement with the fact that the rebinding of BPA hides ferrocenyl groups present in the binding cavities (Figure 2). In the case e-NIP-SPCE, a signal decrease was also observed but to a lower extent, in good correlation with a lower quantity of accessible ferrocenyl groups.

The limit of detection (LOD) was determined from the percentage of current intensity variation of e-MIP-SPCE at $75 \mathrm{mV}$ vs concentration of BPA (Figure 3). The curve obtained revealed a LOQ of $43 \mathrm{ng} / \mathrm{L}$. The comparison with the literature indicates that the proposed e-MIP-SPCE gains a factor of 10 [4].
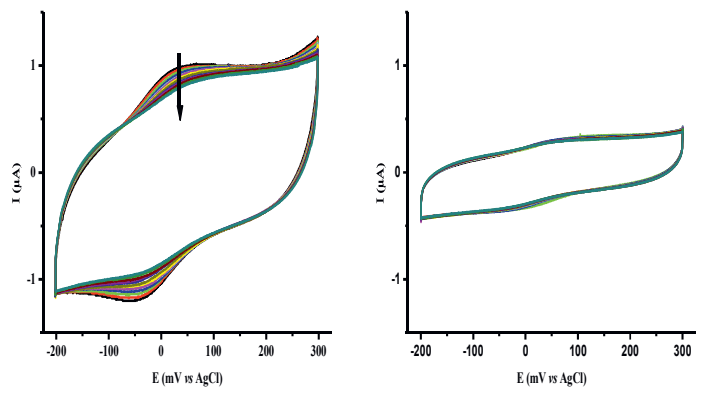

Fig. 2. CV responses of eMIP-SPCE (left) and e-NIP$S P C E$ (right) in $\mathrm{KCl} 0.1 \mathrm{M} /$ water to increasing $B P A$ concentration from 0 to $413 \mathrm{ng} / \mathrm{L}$.

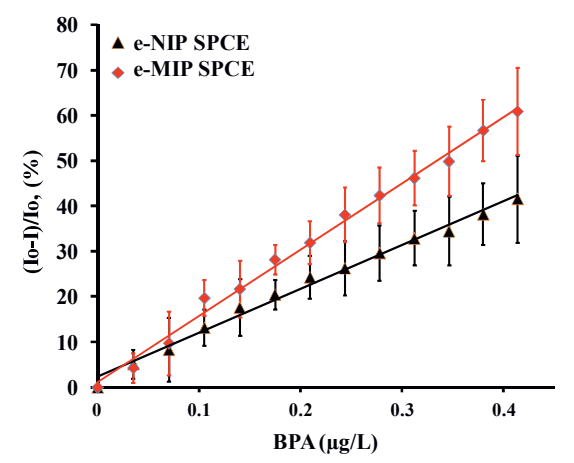

Fig. 3. Percentage decrease of anodic peak current obtained for both polymer-SPCEs.

\section{References}

[1] K. Haupt, K. Mosbach, Molecularly Imprinted Polymers and Their Use in Biomimetic Sensors, Chemical Review 100, 2495-2504 (2000); doi.org/10.1021/cr990099w

[2] D. Udomsap, C. Branger, G. Culioli, P. Dollet, $\mathrm{H}$. Brisset, A versatile electrochemical sensing receptor based on a molecularly imprinted polymer, Chemical Communication 50, 7488-7491 (2014); doi.org/10.1039/C4CC02658F

[3] V. Mba Ekomo, C. Branger, R. Bikanga, A.M. Florea, G. Istamboulie, C. CalasBlanchard, T. Noguer, A. Sarbu, H. Brisset, Detection of Bisphenol $A$ in aqueous medium by screen printed carbon electrodes incorporating electrochemical molecularly imprinted polymers, Biosensors and Bioelectronics, (2018) under press

[4] N. Huang, M. Liu, H. Li, Y. Zhang, S. Yao, Synergetic signal amplification based on electrochemical reduced graphene oxideferrocene derivative hybrid and gold nanoparticles as an ultra-sensitive detection platform for bisphenol A, Anal. Chim. Acta 853, 249-257 (2015); doi.org/10.1016/j.aca.2014.10.016 